\title{
The Potential of TEMPO-Oxidized Nanofibrillar Cellulose Beads for Cell Delivery Applications
}

Renata Aquino, ${ }^{1}$ Gabriella Veronese ${ }^{1}$, Antonio José Felix Carvalho, ${ }^{2}$ Eugen

Barbu, ${ }^{3}$ André Capaldo Amaral ${ }^{1}$ and Eliane Trovatti ${ }^{1}$

${ }^{1}$ Araraquara University Centre, UNIARA, R. Carlos Gomes, 1217, CEP 14801340, Araraquara, SP, Brazil

${ }^{2}$ Department of Materials Engineering, São Carlos School of Engineering, University of São Paulo, Av. João Dagnone 1100, CEP 13563-120, São Carlos, SP, Brazil

${ }^{3}$ School of Pharmacy and Biomedical Sciences, University of Portsmouth, St Michael's Building, White Swan Road, Portsmouth, PO1 2DT, United Kingdom

Corresponding author: elianetrov@yahoo.com.br 
1 Abstract

2 The advanced development of cell carriers for regenerative medicine and cell therapy demands

3 materials able to sustain cell viability prior to their delivery to the target tissue, ability which can

4 be controlled via the shape/size and degradability of the matrix. TEMPO-oxidized nanofibrillar

5 cellulose (ToNFC) macromolecules are negatively charged and therefore can be easily formulated

6 by ionotropic gelation into beads of varying sizes that can release their payload through an

7 erosion-controlled process. We report here for the first time on the preparation of ToNFC beads

8 via ionic gelation using $\mathrm{CaCl}_{2}$ and on their loading with OSTEO-1 rat bone cells, with a view to

9 examine their capacity of sustaining the cell viability and of releasing the bone cells in a controlled

10 manner. The initial results obtained demonstrate that ToNFC is able to protect the OSTEO- 1 cells

11 and to maintain their viability for at least two weeks; following gradual disintegration of the beads,

12 a significant cell release and subsequent proliferation was observed after 7 days. These results

13 indicate the considerable potential of nanofibrillar cellulose (ToNFC) for applications in cell

14 therapy and regenerative medicine.

15

16

17 Key words: cell carriers, TEMPO oxidized cellulose nanofibers, cell delivery,

18 regenerative medicine, bone cells

19

20 
36 Tissue engineering platforms designed for regenerative medicine aim to 37 improve cell viability and engraftment into the damaged organ areas and to promote 38 neoformation of functional tissue. Over the past decade there has been a 39 considerable interest in cell-biomaterial constructs capable to provide an 40 appropriate microenvironment for successful cell transplantation, differentiation 41 and improved cellular functionality. Cell replacement therapy for attaining 42 functional repair requires a biocompatible and biodegradable delivery vehicle 43 capable of successfully maintaining cell viability during transport and of 44 appropriately controlling their release.(Ratner et al. 2014)

45 Several promising novel biomaterial platforms have been developed recently, 46 with natural biomaterials such as proteins or polysaccharides attracting increasing 47 attention for the development of cell carrier materials as many of them are natural 48 components of the extracellular matrix and therefore tend to be biocompatible. 49 However, they also have significant disadvantages such as strict purification 50 requirements to avoid immunogenic responses and inherent source-dependent 51 variability (Willerth et al. 2013); polymers such as fibrin,(Wu et al. 2012) 52 alginate,(Bidarra et al. 2011) or gelatin,(Santoro et al. 2014) represent some of the 53 most studied examples.

54 Among the biomaterials that demonstrate potential for tissue engineering and 55 cell delivery applications, plant-derived materials such as cellulose have received 56 considerable less attention. Cellulose is a biocompatible hydrophilic natural 57 polymer composed solely of glucose monomers and has good mechanical 58 properties (Klemm et al. 2005, Gandini 2011); though it is widely employed in 59 many pharmaceutical/medical applications (native or modified as carboxymethyl 60 cellulose or its ether / ester derivatives), its extremely slow degradation within the 61 human body represents a severe limitation for more complex biomedical 62 applications.

63 Nanofibrillar cellulose (NFC) has attracted however a lot of attention recently 64 due to its high affinity to water and its capability to form highly viscous dispersions 65 at very low solid content; NFC shows particular promise due to its well-defined 66 single component structure and the possibility to finely tune its hydrogel 67 properties.(Bhattacharya et al. 2012, Lin et al. 2014) NFC can be conveniently 
prepared from cellulose using different methods, which lead to nanofibrillar

69 materials with different structural features and properties. One of the alternative

70 methods of separating the native cellulose fibres into nanofibrils is via the

71 controlled 2,2,6,6-tetramethylpiperidine-1-oxyl (TEMPO)-mediated oxidation of

72 the glucose units, (Saito et al. 2007) which generates a cellulose derivative (ToNFC)

73 with increased water solubility due to the high degree of substitution with

74 carboxylic groups (about $90 \%$ ). ToNFC has been investigated for the development

75 of flexible, transparent and biodegradable materials with improved mechanical

76 properties,(Fukuzumi et al. 2009, Jradi et al. 2012) however its potential for

77 applications in the biomedical field (such as in drug delivery) is just starting to be

78 explored.(Weishaupt et al. 2015) What makes ToNFC interesting is that

79 carboxymethyl cellulose derivatives with similar structures have been shown to be

80 resorbable in humans; at physiological $\mathrm{pH}(\sim 7.4)$, about $90 \%$ wt of oxidized

81 cellulose is solubilized within 21 days and converted into the sodium salt of

82 polyglucuronic acid,(Singh et al. 1982) which is then easily eliminated from the

83 body. In ToNFC, the surface of the nanofibrils is negatively charged so a

84 transparent hydrogel made of individual cellulose nanofibers (3-4 nm width, and a

85 few microns length) can be formed.(Saito 2007)

86 Rationalized by the ability of the carboxylic groups present at the surface of

87 the ToNFC nanofibrils to induce their self-assemblage into beads of various sizes

88 based on electrostatic interactions created in the presence of added counter-ions, we

89 report here for the first time on the preparation of OSTEO-1 cell/ToNFC systems.

90 Results of preliminary in vitro investigations of ToNFC beads (particles)

91 formulated as cell carriers that can encapsulate, deliver and then release viable cells

92 for potential tissue engineering applications are described.

\section{Materials and methods}

Dulbecco's modified eagle's medium (DMEM) and fetal bovine serum (FBS) were purchased from Cultilab (Brazil). The 3-(4,5-dimethylthiazol- 2-yl)-2,5-

96 diphenyltetrazolium salt (MTT) was acquired from Merck (US). Isopropyl alcohol

97 dimethyl sulfoxide and phosphate buffer saline (PBS) were purchased from Synth

98 (Brazil); trypsin was sourced from Gibco (US) and 4',6-Diamidino-2-phenylindole

99 (DAPI) from Sigma (Brazil?). All solvents and reagents (analytical grade) were 100 used as purchased. 
ToNFC preparation. Bleached sugar cane bagasse suspension (10 g, $1 \% \mathrm{wt})$,

102 sodium bromide ( $1 \mathrm{mmol} / \mathrm{g}$ cellulose $)$ and TEMPO reagent $(0.1 \mathrm{mmol} / \mathrm{g})$ were

103 stirred together, followed by the slow addition of sodium hypochlorite $(\mathrm{NaClO})(5$

$104 \mathrm{mmol} / \mathrm{g}$ cellulose). The $\mathrm{pH}$ of the suspension was adjusted to 10 by adding $0.1 \mathrm{M}$

$105 \mathrm{NaOH}$. The pulp was then washed with deionized water to neutral $\mathrm{pH}$. The oxidized

106 fibers were sonicated in an ultrasonicator (Hielscher, UP 400S, 400W, 24kHz) until

107 the formation of a transparent gel (about 10min). The carboxylic content was

108 determined by conductometric titration.(Araki et al. 2001) The chemical 109 modification was confirmed by FTIR using a Perkin-Elmer Spectrum 100 FT-IR

110 Spectrometer equipped with a single horizontal Golden Gate ATR cell $\left(4 \mathrm{~cm}^{-1}\right.$

111 resolution, 32 scans).

112 Bone cell culture. Osteogenic cells from the lineage OSTEO-1 (rat calvarium) 113 were incubated in DMEM culture medium supplemented with fetal bovine serum $114(10 \% \mathrm{v} / \mathrm{v})$ and antibiotics (penicillin $100 \mathrm{U} / \mathrm{mL}$; streptomycin $0.1 \mathrm{mg} / \mathrm{mL}$ ). The 115 cells were incubated at $37 \pm 2{ }^{\circ} \mathrm{C}$ in $5 \% \mathrm{CO}_{2}$ atmosphere until they reached 80 to $11690 \%$ confluence, when they were used for the preparation of the beads.

117 Preparation of ToNFC/cell system (beads). All materials were sterilized in an 118 autoclave at $121{ }^{\circ} \mathrm{C}$ for $15 \mathrm{~min}$. For the preparation of the beads, $1 \mathrm{~mL}$ of ToNFC $119(0.8 \% \mathrm{w} / \mathrm{v})$ was gently mixed with the cells pellet obtained by centrifugation $120\left(2.5 \times 10^{4}\right.$ cells $)$. The beads were prepared by drop wise addition of $15 \mu \mathrm{L}$ of ToNFC 121 plus cells mix into a $1.2 \%(\mathrm{w} / \mathrm{v}) \mathrm{CaCl}_{2}$ solution $(50 \mathrm{~mL})$, under magnetic stirring. 122 The beads were separated by filtration using a sieve and used for further 123 measurements as described below.

124 Cell viability measurements. Beads (prepared and isolated as described above) were immersed in the wells of a 96 well microplate filled with DMEM culture medium (two beads/well, each bead was about $5 \mathrm{mg}$ ) and incubated at $37^{\circ} \mathrm{C}$ in a 5

$127 \% \mathrm{CO}_{2}$ atmosphere. The culture media was changed every three days to avoid 128 saturation with cell metabolism products. At each time point $(3,7,10,12$ and 14 129 day, respectively), the ToNFC beads with encapsulated cells were withdrawn and 130 cell viability tests (MTT assay) were carried out for the supernatant media left in 131 each well after the partially eroded beads where removed. MTT reagent $(50 \mu \mathrm{L} ; 0,5$ $132 \mathrm{mg} / \mathrm{mL}$ in PBS) was added to each well and the microplates were protected from 133 light and incubated at $37 \pm 2{ }^{\circ} \mathrm{C}$ for $4 \mathrm{~h}$. The obtained formazan crystals were 
134 solubilized in isopropyl alcohol (100 $\mu \mathrm{L}$ per well) and the absorbance for each well

135 was read at $570 \mathrm{~nm}$ in an ELISA microplate reader Polaris-Celer

136 spectrophotometer. The viability assays were performed in three independent runs,

137 in different days, each run being done in triplicate. The cell viability was reported

138 as optical density (O.D.).

139 SEM studies. The morphology of the released (adhered) cells was assessed 140 using a FEI (XL50, Philips) Scanning Electron Microscope. Cells were fixed with

141 glutaraldehyde solution $(2.5 \%)$ for $40 \mathrm{~min}$ at $8{ }^{\circ} \mathrm{C}$, then washed with distilled water,

142 freeze-dried (Liotop L 101) and coated with evaporated carbon in a sputter (Q150R

143 Quorum Technologies, UK) before being examined by SEM. Optical microscopy

144 was carried out using a Leica DM 750 digital microscope equipped with a

145 fluorescence accessory ( $\mathrm{Hg}$ lamp). For examining the cells, the beads were

146 immersed into $1 \mu \mathrm{m} / \mathrm{mL}$ DAPI solution for ten minutes and then were analysed by

147 fluorescence microscopy.

\section{Results and discussion}

Preparation of the ToNFC beads. ToNFC was synthesised via a controlled 2,2,6,6-tetramethylpiperidine-1-oxyl (TEMPO)-mediated oxidation of the C6

151 primary hydroxyl groups of the glucose units, following a previously reported

152 method (Saito 2007). The chemical modification of the cellulosic substrate was

153 confirmed by FTIR-ATR (results presented as supplementary information). The

154 content of carboxylic groups was found to be $1.1 \mathrm{mmol} \mathrm{g}^{-1}$, as determined by

155 conductometric titration. While ToNFC is normally used as a film or gel, here we

156 investigated the assembly of ToNFC as beads in the presence of $\mathrm{Ca}^{2+}$ ions (Figure

157 1A) via an ionic gelation process typical for anionic polymers such as alginates

158 (Ostberg et al. 1994); to the best of our knowledge, this is the first report on the

159 preparation of ToNFC beads. The size of the beads can be controlled during the

160 preparation stage by varying the volume of the ToNFC suspension added (Figure

161 1B); beads of about $2 \mathrm{~mm}$ in diameter were used for further experiments. 
164
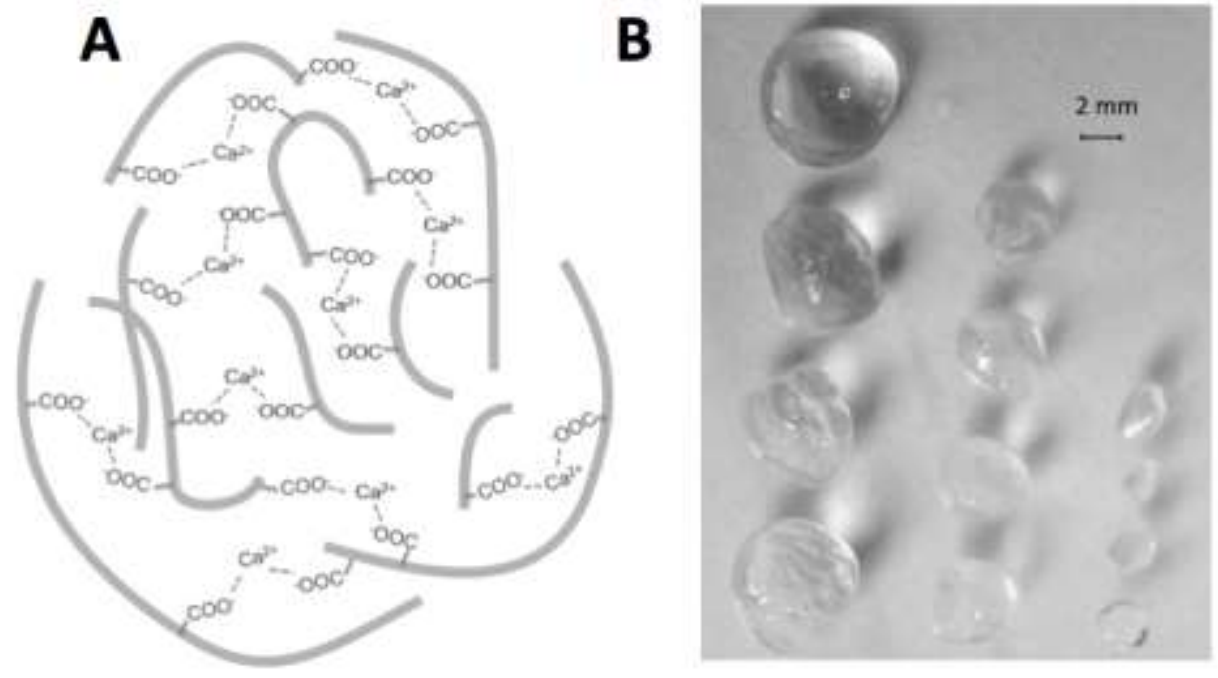

165 Figure 1. (A) A schematic representation of the electrostatically-crosslinked network generated as

166 a result of the strong interactions between ToNFC carbolxilix groups and $\mathrm{Ca}^{2+}$ ions. (B) An image

167 of ToNFC beads of different diameter, obtained using either $100 \mu \mathrm{L}, 50 \mu \mathrm{L}$, or $15 \mu \mathrm{L}$ of ToNFC

168 suspension $(0,8 \% \mathrm{w} / \mathrm{v})$, respectively (left to right).

169 Cell encapsulation into ToNFC. The incorporation of rat bone cells within the

170 ToNFC beads was successfully carried out as above, using a suspension composed

171 of cells and ToNFC instead of just ToNFC solution. Optical microscopy images of

172 ToNFC beads with encapsulated cells are presented in Figure 2, where the cells

173 located close to the surface are clearly visible (Fig. 2 A) and their presence

174 confirmed by nucleus staining with DAPI (Fig. 2B). 


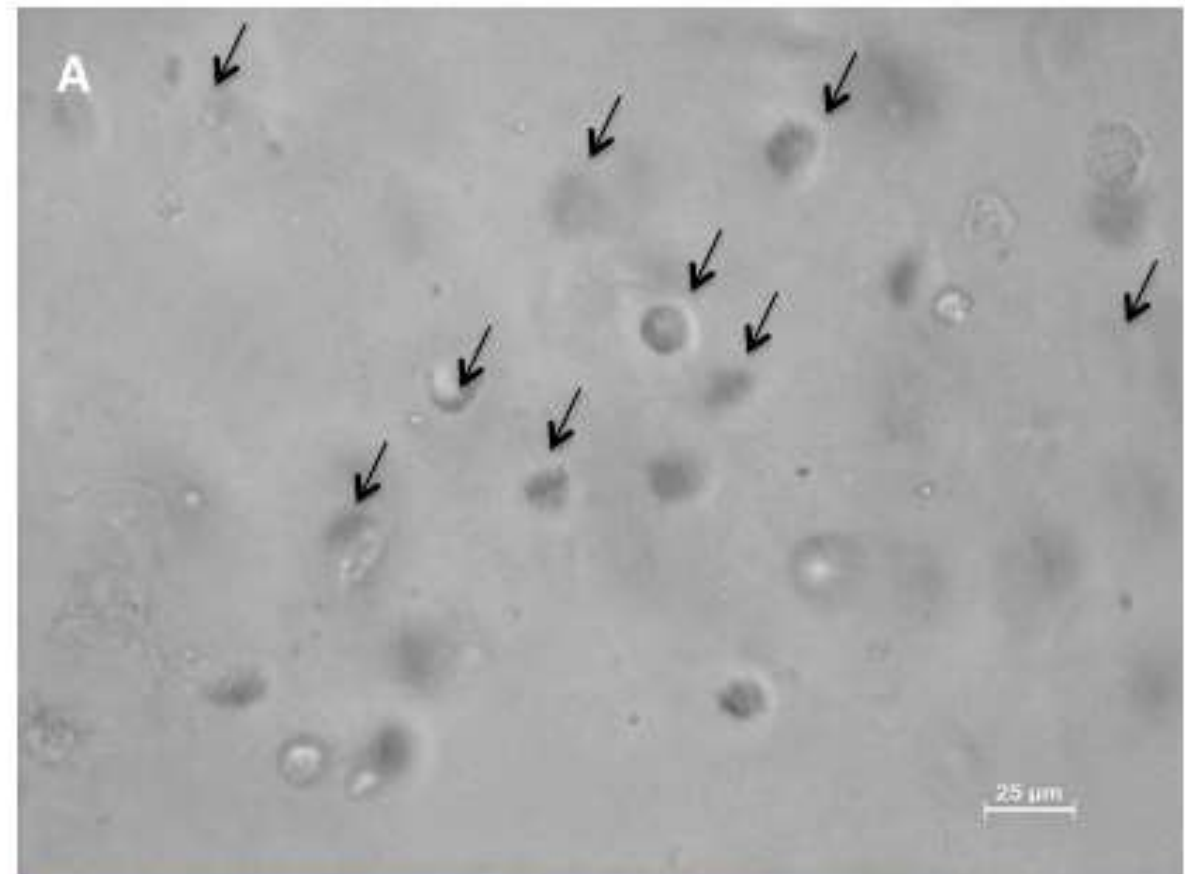

\section{B}

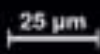

178 Figure 2. (A) ToNFC beads with encapsulated cells indicated by arrows; (B) cell presence

179 confirmed by DAPI staining (blue dichroic filter).

180 Cell viability. Measurements of cell viability were carried out using an MTT

181 assay that is indicative of the mitochondrion dehydrogenase activity (MTT is

182 reduced by NADH to a purple colored formazan salt that act as a quantifiable

183 marker for living cells only). Results expressed as O.D. (Figure 3) indicated no cell

184 proliferation for at least one week as the result of their protective entrapment within

185 the ToNFC based matrix. At the $7^{\text {th }}$ day time point, the results of MTT indicated an 
186 O.D. value of $0.048( \pm 0.00)$, which increased dramatically reaching $1.01( \pm 0.20)$

187 at day 14.

188

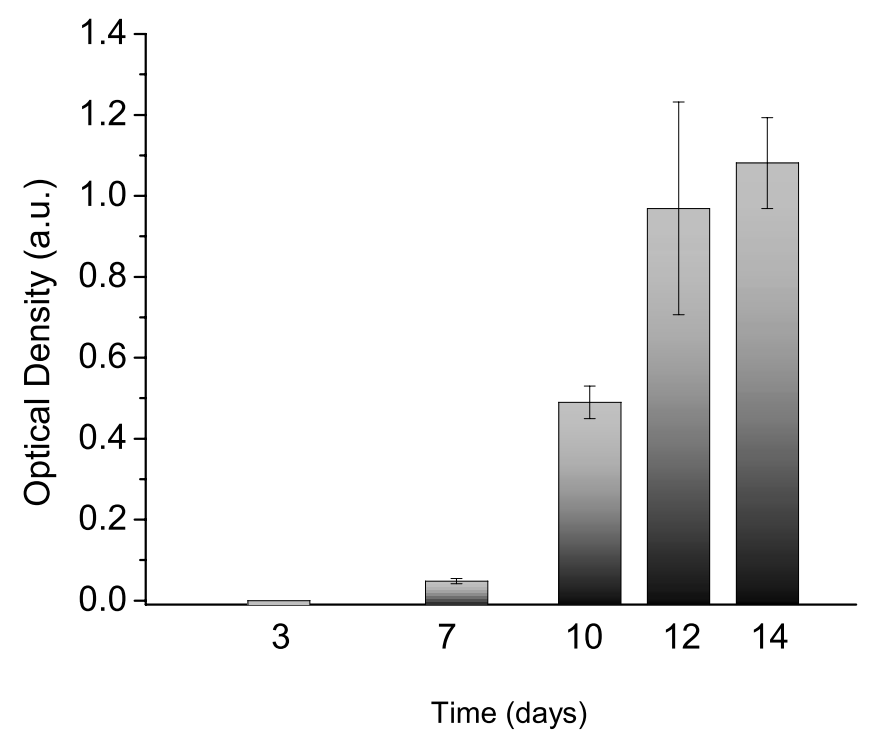

189 Figure 3 - Results of MTT tests indicative of the released cell viability.

190 In addition to the positive MTT results, at the 7 day time point it was possible 191 to visualise sparse cells that became adhered to the bottom of the microplate well 192 (Figure 4 A). The morphology of the adhered cells indicated their spreading, 193 indicative as well of their viability and in agreement with the MTT results (the low

194 number of adhered cells indicated they had just started to proliferate). After 14 days,

195 a confluent cell monolayer with polygonal morphology was observed at the bottom 196 of the microplate wells (Fig. 4 B). It is important to note that the cells were 197 increasingly released into the medium following the gradual erosion/disintegration 198 of the beads. Their slow release was noticeable from day 7 and eventually led to the 199 increased viability detected by MTT at day 14, when most beads were completely 200 fragmented (with the released cells capable to adhere to the bottom of the 201 microplate and even to start proliferating). 

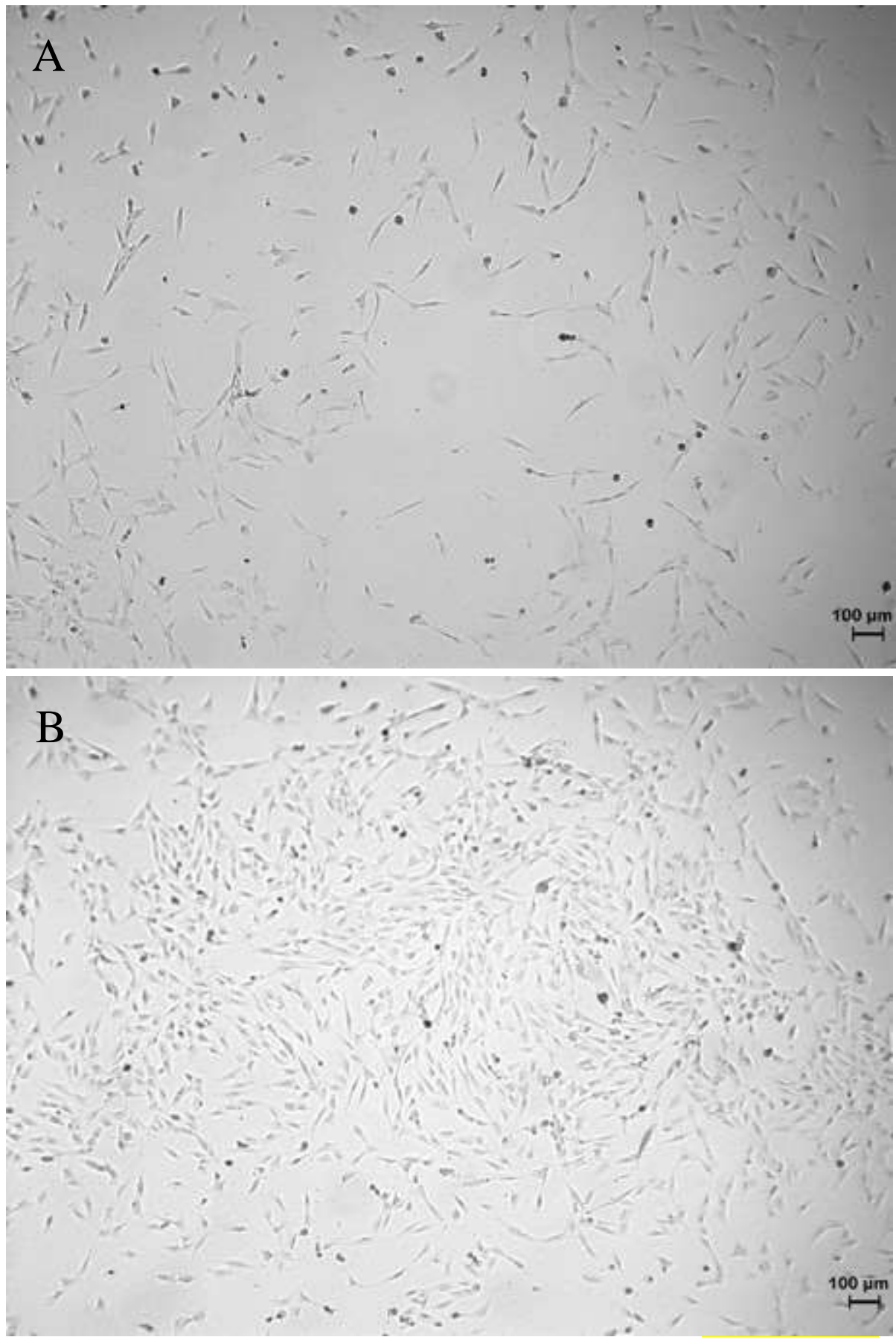

203 Figure 4 - Images of cell adhered at the bottom of the microplate wells: (A) after 7 days; (B) after 20414 days.

SEM images of the cells adhered at the surface of ToNFC after $24 \mathrm{~h}$ of 206 encapsulation are presented in Figure 5. The arrows in Figure 5B point to cells 207 present at the surface of ToNFC and the magnified image in Figure 5D shows the 208 morphology of a surface spread cell (Fig. 5C), indicating its viability in contact with 209 ToNFC. 


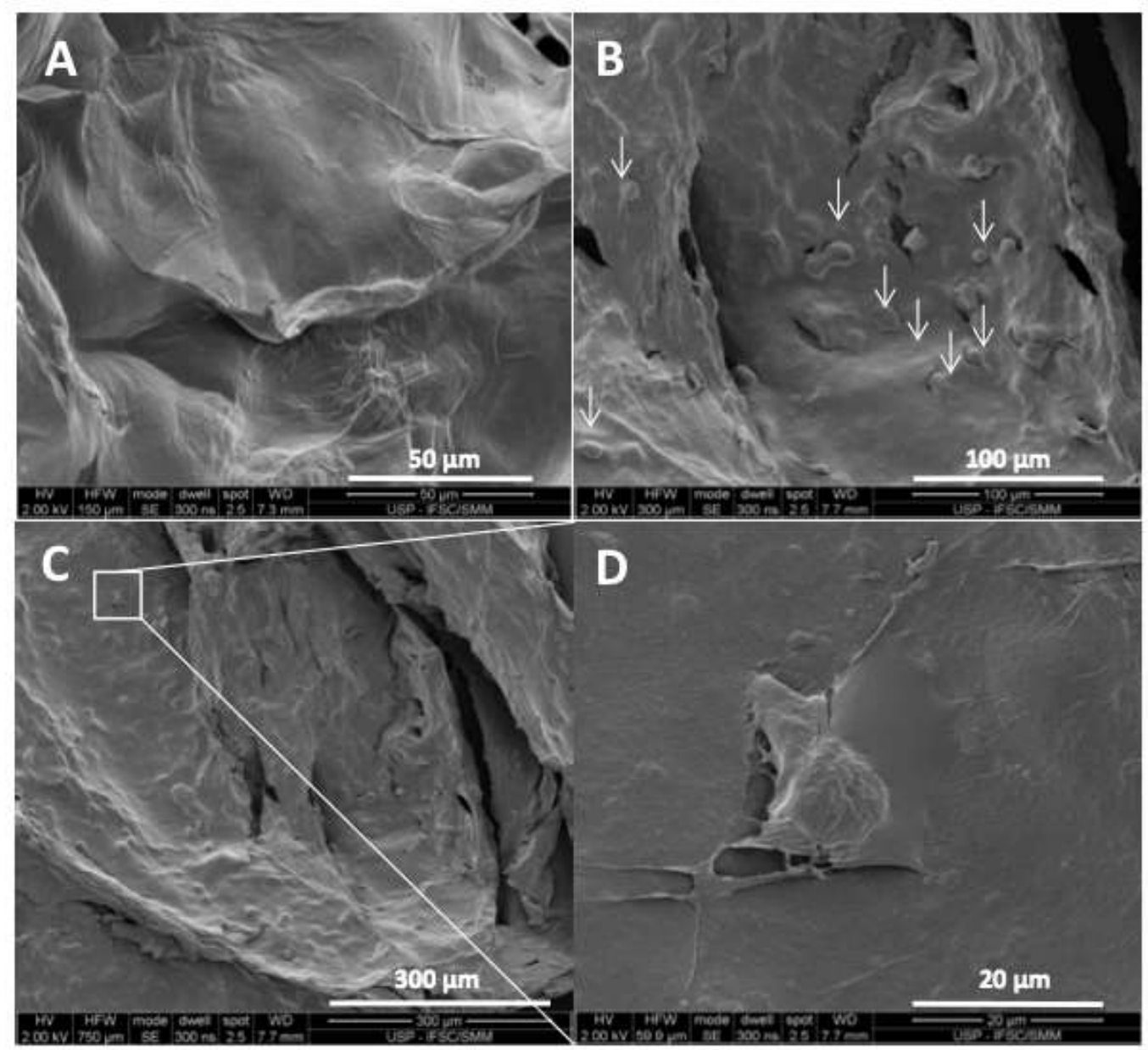

212 Figure 5. SEM images of ToNFC beads with and without OSTEO- 1 cells after $24 \mathrm{~h}$ incubation

213 time: (A) no cells; (B, C and D) with cells (different magnification).

\section{Erosion and degradation of ToNFC beads.}

217 applications are their insolubility and the lack of biodegradation in the human body,

218 explained by the absence of specific enzymes required for this process

219 (cellulases).(Lin 2014) In the case of our ToNFC beads, it appears the degradation

220 observed is a physical process controlled by the gradual loss of $\mathrm{Ca}^{2+}$ ions due to

221 their diffusion into the aqueous medium into which the beads were incubated,

222 leading to $\mathrm{Ca}^{2+}$ ion concentration gradient. The time the beads take to disintegrate

223 is an important parameter, as in such applications the carrier material has to deliver

224 the cells well into the target tissue in order to increase the amount of cells required

225 to effect the anticipated regeneration and decrease the healing time. The

226 experiments showed that cells started to adhered to the plate at day 7 , indicating 
227 that beads needed about a week to noticeably begin disintegrating (leading to the

228 formation of small debris in suspension) and releasing the cells. The results

229 emphasize important advantages of ToNFC compared to unmodified cellulose,

230 namely the possibility to form electrostatically cross-linked hydrogels with better

231 mechanical properties and significantly higher capacity to degrade.

\section{Conclusions}

ToNFC was formulated via ionic gelation with $\mathrm{Ca}^{2+}$ ions into beads that were

234 loaded with OSTEO-1 cells; the size of the ToNFC beads and the cell content can

235 be easily controlled at the preparation stage. The hydrophilic nature of the ToNFC

236 matrix allowed water and nutrient access to the OSTEO-1 cell payload and

237 contributed to maintaining cell viability, as demonstrated by the fast proliferation

238 observed following the release. We believe that the potential to maintain cell

239 viability and to release the cell cargo by physically-induced degradation

240 demonstrated by the TEMPO-oxidized cellulose fibres that were formulated into

241 beads via ionic gelation warrants further detailed investigations.

\section{Acknowledgments}

The authors thank Fapesp, CNPq and UNIARA University Centre for funding the projects related to the paper. G. V. thanks UNIARA University Centre for the undergraduate scholarship.

\section{References}

Araki, J (2001). Steric stabilization of a cellulose microcrystal suspension by poly(ethylene glycol) grafting. Langmuir 17 21-27.10.1021/la001070m Bhattacharya, M (2012). Nanofibrillar cellulose hydrogel promotes three-dimensional liver cell culture. Journal of Controlled Release 164 291-298.10.1016/j.jconrel.2012.06.039

Bidarra, S J (2011). Injectable in situ crosslinkable RGD-modified alginate matrix for endothelial cells delivery. Biomaterials 32 7897-7904.10.1016/j.biomaterials.2011.07.013

Fukuzumi, H (2009). Transparent and High Gas Barrier Films of Cellulose Nanofibers Prepared by TEMPO-Mediated Oxidation. Biomacromolecules 10 162-165.10.1021/bm801065u macromolecular science and technology. Green Chemistry 13 1061-1083 
Ostberg, T (1994). Calcium Alginate Matrices for Oral Multiple-Unit Administration .3. Influence of Calcium-Concentration, Amount of Drug Added and Alginate Characteristics on Drug-Release. International Journal of Pharmaceutics 111 271-282.Doi 10.1016/0378-5173(94)90350-6

Ratner, B D (2014). Biomaterials Science: An Introduction to Materials in Medicine. Oxford, UK, Elsevier Inc. .

Saito, T (2007). Cellulose nanofibers prepared by TEMPO-mediated oxidation of native cellulose. Biomacromolecules 8 2485-2491.10.1021/bm0703970

Santoro, M (2014). Gelatin carriers for drug and cell delivery in tissue engineering. Journal of Controlled Release 190 210-218.10.1016/j.jconrel.2014.04.014Singh, M (1982). Biodegradation Studies on Periodate Oxidized Cellulose. Biomaterials 3 16-20Weishaupt, R (2015). TEMPOOxidized Nanofibrillated Cellulose as a High Density Carrier for Bioactive Molecules. Biomacromolecules 16 3640-3650.10.1021/acs.biomac.5b01100Willerth, S M (2013). Combining stem cells and biomaterial scaffolds for constructing tissues and cell delivery Stem Book. D. Melton. Harvard Stem Cell Institute, Massachusetts. Massachusetts General Hospital.Wu, X W (2012). Fibrin glue as the cell-delivery vehicle for mesenchymal stromal cells in regenerative medicine. Cytotherapy 14 555-562.10.3109/14653249.2011.638914

\section{Supplementary Information}

The chemical modification of the cellulosic substrate was confirmed by FTIR-ATR, as showed in Figure S1 for nanofibers of cellulose obtained from sugar cane bagasse (NFC) and for ToNFC. For both samples, the FTIR spectra revealed the typical features of cellulosic substrates, with strong bands at around 3300, 2880, and $1100 \mathrm{~cm}^{-1}$ associated with the vibrations of the $\mathrm{O}-\mathrm{H}, \mathrm{C}-\mathrm{H}$, and $\mathrm{C}-\mathrm{O}-\mathrm{C}$ groups, respectively. ToNFC spectra showed the strong peak at $1596 \mathrm{~cm}^{-1}$ attributed to the asymmetric stretching vibration of $\mathrm{COO}^{-}$, thus confirming the chemical modification of cellulose.

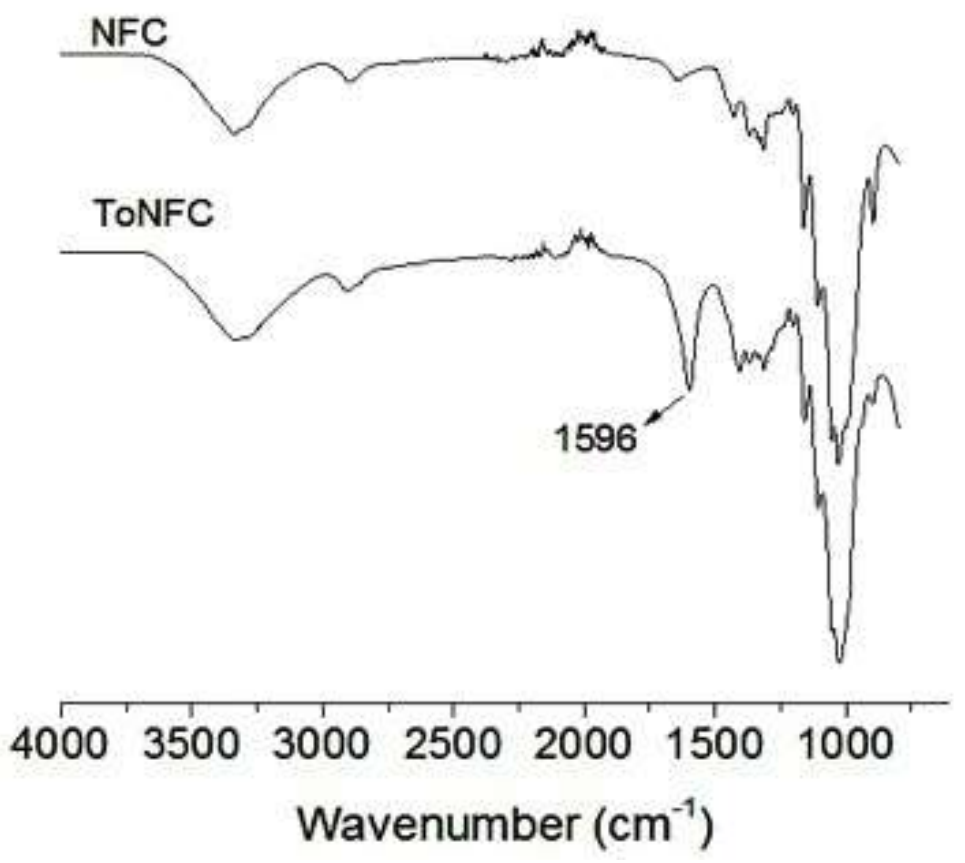

291 Figure S1. FTIR-ATR spectra of sugar cane nanofibers and its derivative ToNFC.

292 The nanometric dimension of ToNFC as obsesrved by SEM (Figure 2) also supports the intended 293 chemical modification of the sugar cane bagasse. 


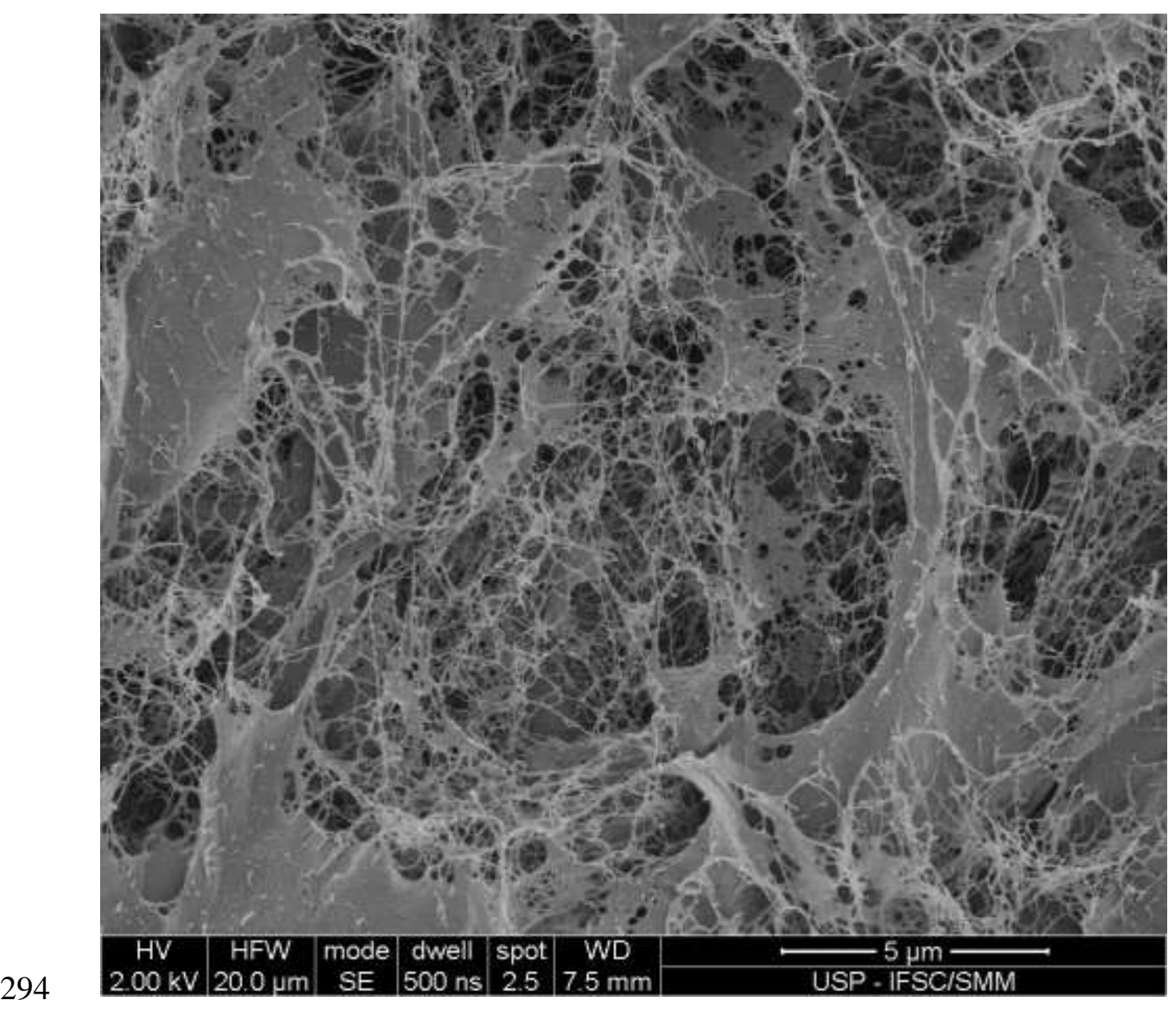

295 Figure S2. SEM image of the surface of freeze dried ToNFC (no cells).

297 Additional optical microscopic images of empty beads and beads with encapsulated 298 cells are showed in Figure S3. Images of beads with encapsulated cells before and 299 after staining with DAPI are presented in Figure S4. 

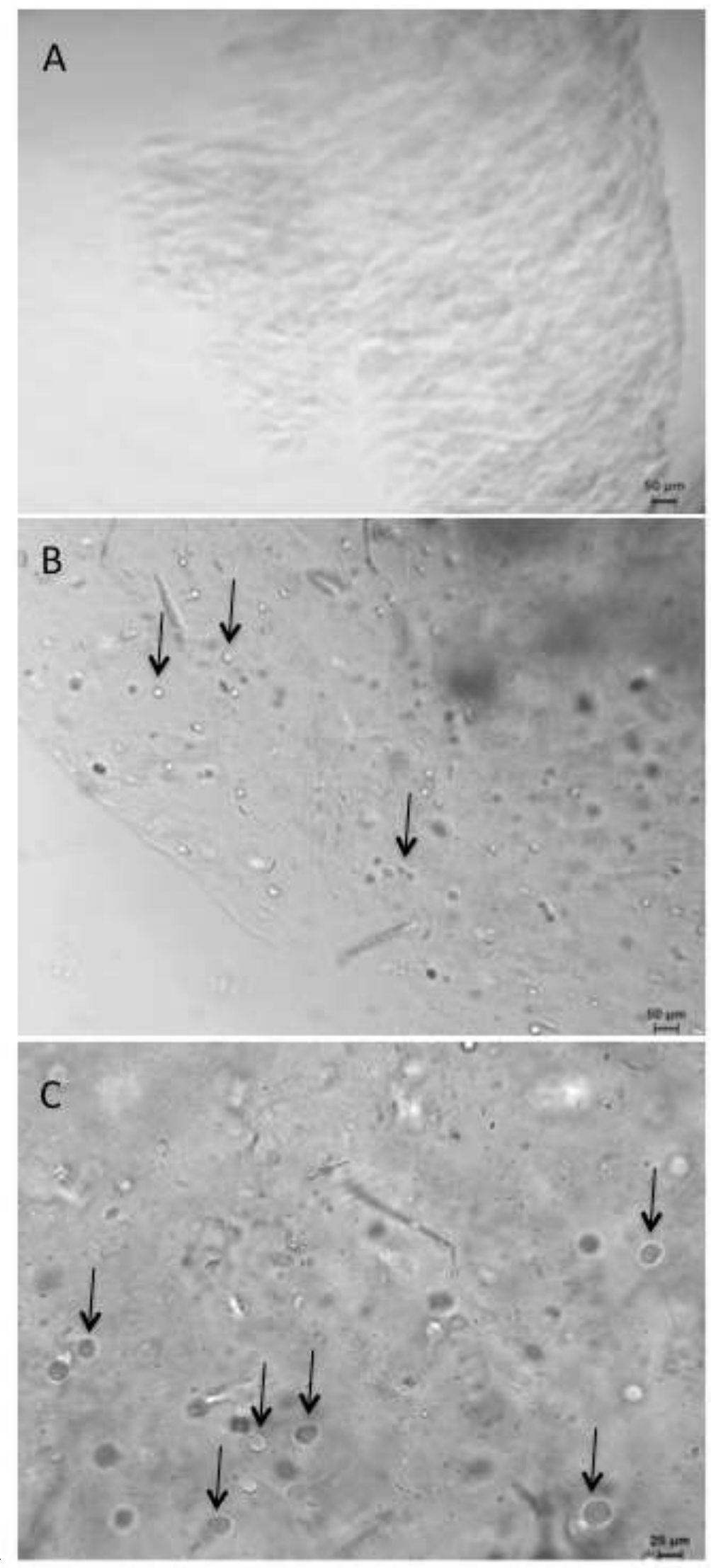

311 Figure S3. (A) Empty ToNFC beads; (B) ToNFC beads with encapsulated cells indicated by arrows, 312 scale bar $50 \mu \mathrm{m}$; (C) ToNFC beads with encapsulated cells indicated by arrows, scale bar $25 \mu \mathrm{m}$. 


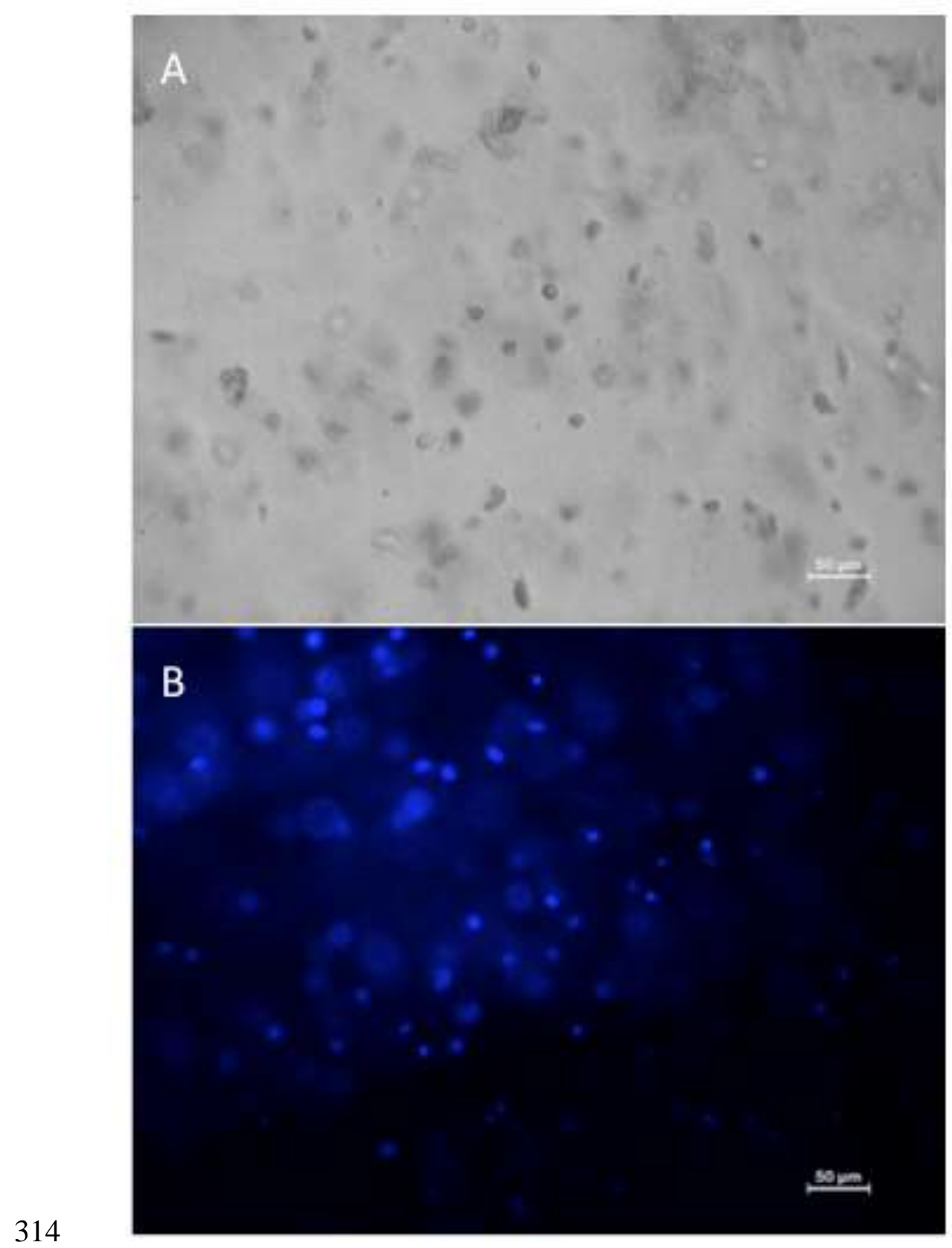

315 Figure S4. ToNFC beads with encapsulated cells before (A) and after staining with DAPI (B). Scale 316 bars $50 \mu \mathrm{m}$. 